\title{
Discovery of Likely Globular Clusters in Maffei 1
}

\author{
R. Buta \\ Department of Physics and Astronomy, University of Alabama, Box \\ 870324, Tuscaloosa, AL 35487, USA \\ M. L. McCall \\ Department of Physics and Astronomy, York University, 4700 Keele \\ Street, North York, ON M3J 1P3, Canada
}

\begin{abstract}
The Hubble Space Telescope Wide Field and Planetary Camera 2 was used to image at high resolution the core region of the nearby, heavily obscured massive elliptical galaxy Maffei 1 . We report on the discovery of 19 diffuse objects in the WFPC2 field that are likely to be globular clusters associated with Maffei 1 . We present some preliminary data on the luminosities, colors, and sizes of these candidates.
\end{abstract}

\section{Introduction}

Maffei 1 is a heavily-obscured galaxy located in the Zone of Avoidance near the large Galactic HII region IC 1805 (Maffei 1968). It is one of the dominant members of the IC 342/Maffei Galaxy Group, and is the nearest example of a normal intermediate-to-giant elliptical galaxy (Buta and McCall 1999 and references therein).

Recently, Maffei 1 was imaged in the filters F675W ( $R$-band) and F814W ( $I$ band) with the WFPC2 on the Hubble Space Telescope to study its core region at high resolution. Dithering was used to improve sampling of the point spread function, and the nucleus was centered on the PC1 frame. In the surrounding WF2-4 frames, 19 slightly diffuse objects have been identified. We suggest that most of these objects are globular clusters associated with Maffei 1. The WFPC2 images have been used to extract magnitudes and colors for the candidates in order to compare them with Galactic globular clusters. Candidates were identified only on the basis of size and not color (Figure 1).

The Galactic extinction towards Maffei 1 is high, about $5 \mathrm{mag}$ in the $V$ band according to Buta and McCall (1983). For our preliminary analysis of the candidate clusters, we use $A_{F 675 W}=3.6 \mathrm{mag}$ and $A_{F 814 W}=2.7 \mathrm{mag}$, based on $E(B-V)=1.5 \mathrm{mag}$ from Buta and McCall (1983), and using Table $12 \mathrm{~b}$ of Holtzman et al. (1995). We also adopt the distance, $4.2 \mathrm{Mpc}$, derived by Luppino and Tonry (1993) from near-infrared surface brightness fluctuations. We are currently re-evaluating both the distance and extinction of Maffei 1 using recently-obtained spectroscopic and photometric data (Fingerhut et al. 2001; Buta \& McCall 1999). 


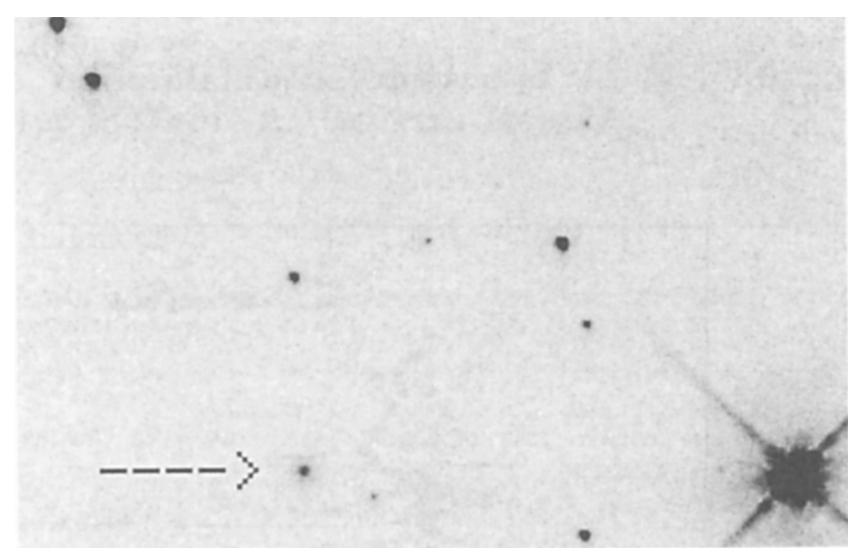

Figure 1. Small part of WFPC2 field (filter F814W) showing the brightest candidate globular cluster (arrow). The field lies approximately $100^{\prime \prime}$ south of the center of Maffei 1 . The arrow is 4 "' 25 in length. North is approximately to the upper right, and east is approximately to the upper left.

\section{Luminosities, Colors, and Sizes of Candidate Clusters}

Figure 2 shows a plot of extinction-corrected absolute magnitude $M_{I}^{0}$ versus extinction-corrected color $(R-I)_{0}$ for all 19 candidates as compared to a sample of Galactic globular clusters compiled by Harris (1996). Only five of the 19 candidates appear to lie more than $1 \sigma$ in $(R-I)_{0}$ outside the region occupied by Galactic globulars. Some of the scatter in $(R-I)_{0}$ could be due to variable extinction, which appears especially significant to the north and west of the nucleus of Maffei 1. The apparently brightest candidate cluster (Figure 1) has a corrected absolute magnitude of $M_{I}^{0}=-10.4$ and a corrected color index of $(R-I)_{0}=0.53 \pm 0.04$, both comparable to M3. Note that we cannot rule out at this time that some of the candidates might be background galaxies. However, none of the diffuse objects shows an elongated shape that might be indicative of a background galaxy.

The growth curves of the three brightest candidates were fitted with an analytic King model convolved with the point spread function, approximated for this preliminary study by a gaussian. These candidates are found to have core radii and concentration parameters $\left(r_{c}, c\right)$ of $(1.5 \mathrm{pc}, 1.80),(1.3 \mathrm{pc}, 1.05)$, and $(3.3 \mathrm{pc}, 1.50)$, comparable to Galactic globulars such as M3, NGC 6333, and NGC 7006, respectively. However, the absolute magnitudes are 0.5-1.3 mag brighter than the listed Galactic globulars, perhaps because of a selection effect due to the high extinction, or because of an overestimate of the distance.

The discovery of likely globular clusters in Maffei 1 provides us with a new nearby system of clusters that potentially can be studied in great detail in the near-infrared. The full analysis of our WFPC2 data, including our study of the core region of Maffei 1, will be contained in Buta \& McCall (2001). RB 


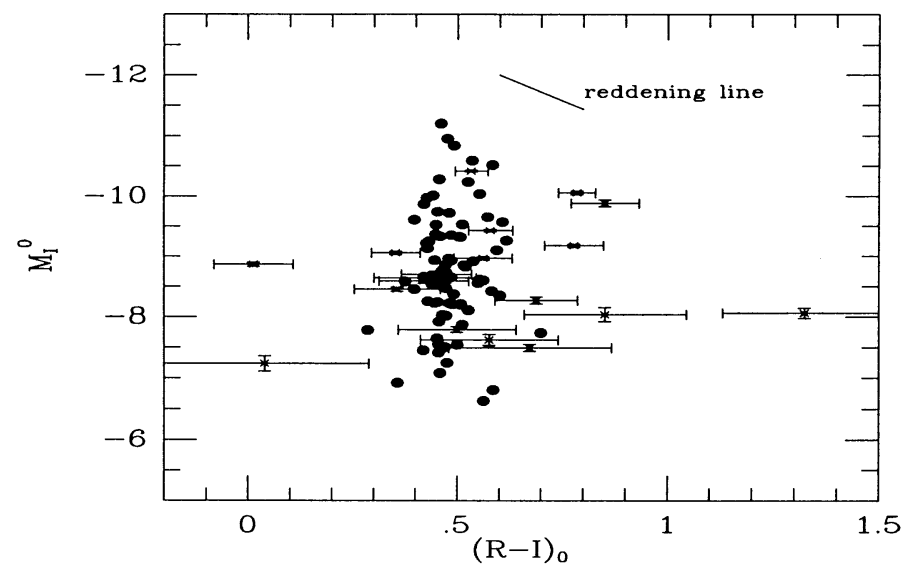

Figure 2. Color-Magnitude Diagram of 19 Candidate Maffei 1 clusters (crosses with error bars), as compared to Galactic globular clusters (filled circles, from Harris 1996). The Maffei 1 objects are corrected for $3.6 \mathrm{mag}$ in $R$ and $2.7 \mathrm{mag}$ in $I$. Error bars are larger in $R$ due to a shorter exposure time and the higher extinction compared to $I$. Variable extinction may also contribute to the scatter in $(R-I)$ colors for the cluster candidates.

acknowledges the support of NASA/STScI Grant GO 8101. MLM acknowledges the support of the National Research Council of Canada.

\section{References}

Buta, R., \& McCall, M. L. 1999, ApJS, 124, 33

Buta, R., \& McCall, M. L. 2001, in preparation

Buta, R., \& McCall, M. L. 1983, MNRAS, 205, 131

Fingerhut, R. L., McCall, M. L., DeRobertis, M. M., Kingsburgh, R. L., Kolmjenovic, M., Lee, H., Ross, R. \& Buta, R. J. 2001, in preparation

Harris, W. E. 1996, AJ, 112, 1487

Holtzman, J. et al. 1995, PASP, 107, 1065

Luppino, G. \& Tonry, J. L. 1993, ApJ, 410, 81

Maffei, P. 1968, PASP, 80, 618 